\title{
Phononic crystal as a homogeneous viscous metamaterial
}

\author{
M. Ibarias, ${ }^{1}$ Yu. Zubov, ${ }^{2}$ J. Arriaga, ${ }^{1}$ and A. A. Krokhin $\odot^{2, *}$ \\ ${ }^{1}$ Instituto de Física, Universidad Autónoma de Puebla, Apartado Postal J-48, 72570, Puebla, México \\ ${ }^{2}$ Department of Physics, University of North Texas, P. O. Box 311427, Denton, Texas 76203, USA
}

(Received 16 September 2019; accepted 18 May 2020; published 8 June 2020)

\begin{abstract}
A homogenization theory, representing the low-frequency limit, is developed for a phononic crystal of cylinders embedded in a viscous fluid. The decay coefficient of sound due to viscosity is calculated analytically for any two-dimensional Bravais lattice and cross section of the cylinders. It is shown that due to the formation of a viscous boundary layer around each cylinder, the losses are enhanced by two to three orders of magnitude as compared to the losses in the free fluid. Also, the decay coefficient in a phononic crystal scales with frequency as $\sqrt{\omega}$, unlike $\omega^{2}$ scaling known for free viscous fluid. In the low-frequency limit a phononic crystal with asymmetric unit cell behaves like a dissipative homogeneous metafluid with anisotropic viscosity.
\end{abstract}

DOI: 10.1103/PhysRevResearch.2.022053

Introduction. Pressure in a plane sound wave propagating in a viscous homogeneous fluid decays exponentially with distance, $p(x) \sim e^{-\gamma_{0} x}$. The decay coefficient $\gamma_{0}=\frac{\omega^{2}}{2 \rho c^{3}}\left[\frac{4}{3} \eta+\xi\right]$ grows quadratically with frequency $\omega$ [1]. It depends on fluid density $\rho$, two viscosity coefficients $\eta$ and $\xi$, and speed of sound $c$. The decay length of sound in water at the frequency of $50 \mathrm{kHz}$ is $1 / \gamma_{0} \approx 15 \mathrm{~km}$. Such slow decay means that dissipative losses can be ignored in free water. However, they are strongly increased if sound wave meets a solid object on its way. Oscillating fluid sticks to solid boundaries, forming a narrow viscous layer of thickness $\delta=\sqrt{2 \eta /(\omega \rho)}$ (so-called Stokes boundary layer) where velocity gradients greatly exceed the gradients in a free fluid, leading to much higher viscous losses. The effect of enhanced losses for reflection from a solid boundary was predicted by Konstantinov [2] (see also Ref. [3]). Viscous losses for reflection grow as $\sqrt{\omega}[1,4]$.

In a phononic crystal, the volume occupied by the viscous boundary layers formed around solid scatterers usually constitutes a small part of the volume of the whole sample. It, however, strongly reduces acoustic transmission. Almost $30 \%$ reduction of transmission through a metasurface was registered while the boundary layer was less than $3 \%$ of the metasurface thickness [5]. Strong absorption within the boundary layer not only reduces acoustic transmission but also affects the speed of sound, in spite of the fact that the principal part of the wave front propagates far away from the solid-fluid boundaries where fluid is practically inviscid [6]. While the fraction of viscous fluid in a phononic crystal is reduced by a factor $1-f$ as compared to $100 \%$ in a free fluid ( $f$ is the filling factor of solid inclusions), a sound wave

\footnotetext{
*arkady@unt.edu
}

decays much faster in phononic crystal due to the Konstantinov effect. In a phononic crystal designed for manipulation of sound, the decay coefficient $\gamma_{\mathrm{ph}}$ lies within the interval $\gamma_{0} \ll$ $\gamma_{\mathrm{ph}} \ll 1 / a_{0}$, where $a_{0}$ is the period of phononic crystal. The latter inequality allows perturbative treatment of the viscosity effects and calculation of $\gamma_{\mathrm{ph}}$ in the lowest approximation over $\xi$ and $\eta$. In this approximation other effective parameters, like speed of sound, elastic modulus, etc, turn out to be viscosityindependent and coincide with their values obtained for the corresponding inviscid fluid [7]. Dependence on viscosity appears in the higher approximations over $\eta$.

In a steady flow of viscous fluid through a periodic arrangement of solid inclusions a traction of the fluid on each inclusion defines the effective steady-state viscosity. A rigorous theory was developed in Ref. [8]. It was shown there that in general case the effective velocity is a fourth-rank tensor. Numerical results obtained for three cubic lattices of spheres demonstrate that the effective viscosity grows with filling fraction $f$. For the values of $f$ near close-packing the effective viscosity increases by order of magnitude as compared to the viscosity of the fluid. It will be shown here that fast oscillations of fluid in a sound wave give rise to much stronger increase of dynamic viscous losses.

A qualitative estimate for the decay coefficient $\gamma_{\mathrm{ph}}$ of sound in a periodic structure caused by viscous losses can be obtained from the well-known formula [1,4] for the relative energy loss at reflection of sound with wavelength $2 \pi / k$ from a hard flat boundary, $\Delta E / E \sim \delta k / 2 \pi \sim c^{-1} \sqrt{\omega \eta / \rho}$. If sound propagates through a periodic lattice of cylindrical rods embedded in a viscous fluid, then the dissipation occurs within the boundary layer formed over each scatterer. If the circumference of the scatterer is $L_{0}$, then the dissipative loss within a unit cell is $\Delta E / E \sim(\delta k / 2 \pi)\left(L_{0} / a_{0}\right) \sim\left(L_{0} / a_{0} c\right) \sqrt{\omega \eta / \rho}$. The decay coefficient is the energy loss per unit length, i.e.,

$$
\gamma_{\mathrm{ph}}=\frac{\Delta E}{a_{0} E} \sim \frac{1}{c a_{0}} \sqrt{\frac{f \omega \eta}{\rho}} .
$$


Here $f \sim\left(L_{0} / a_{0}\right)^{2}$ is the filling fraction of hard scatterers in 2D lattice [9].

According to Eq. (1) the decay length of $50 \mathrm{kHz}$ sound in a phononic crystal $(f \sim 0.5)$ of hard scatterers in water is about $1 / \gamma_{\mathrm{ph}} \sim 200 \mathrm{~m}$, i.e., it is reduced by two orders of magnitude as compared to free water. Equation (1) does not take into account multiple scattering and possible interference and diffraction. Therefore Eq. (1) gives the attenuation coefficient for irregular structure consisting of single scatterers if the filling fraction $f$ is not very small, $L_{0} \sim a_{0}$. The problem of acoustic scattering at a single elastic cylinder in a viscous fluid environment was solved in Ref. [10]. The value of the attenuation coefficient at $50 \mathrm{kHz}$ extracted from Fig. 8 of Ref. [10] is approximately $6 \times 10^{-4} \mathrm{~dB} / \mathrm{cm}$. For free water it is $7 \times 10^{-6} \mathrm{~dB} / \mathrm{cm}$, i.e., two orders of magnitude less. Thus, Eq. (1) estimates correctly the enhanced viscous losses in a phononic crystal in a simple case of isotropic lattice and noninteracting scatterers. Square-root scaling of the attenuation coefficient with frequency was measured for a periodic array of narrow slits in air [11]. The exact solution obtained in Ref. [10] and Eq. (1) confirm that dissipative losses within the viscous boundary layer significantly exceed the losses in the bulk of fluid and, therefore, the latter can be neglected. Here we develop an analytical approach, for exact calculations of the attenuation coefficient in a two-dimensioonal (2D) phononic crystal in the low-frequency limit. The exact results show that due to multiple scattering and particular shape of the cross section of the cylinders the dissipative losses get further enhancement. In a periodic structure the decay coefficient may exceed by one-two orders of magnitude the enhancement predicted by Eq. (1) and obtained in Ref. [10] for a single scatterer.

Dissipation of acoustic energy around a hard scatterer. Exact analytical results for $\gamma_{\mathrm{ph}}$ can be obtained in the lowfrequency limit when the field of pressures and velocities produced by sound can be calculated explicitly. In the longwavelength limit a phononic crystal behaves, in many cases, like a homogeneous medium with effective parameters. The effective parameters for different types of phononic crystals have been calculated in a series of recent publications [12-25]. Deformation of photonic and phononic crystal spectra and, in particular, the band-gap width under the influence of weak dissipation was studied in Refs. [26-30]. The dissipation was introduced phenomenologically by adding a frequencydependent imaginary part to the dielectric or elastic coefficients.

In phononic crystals of solid-fluid constituents the dissipation of sound of frequencies less than $1 \mathrm{MHz}$ occurs mainly in the viscous fluid. Viscous losses depend on the distribution of velocities in the fluid, which is very sensitive to the shape and mutual orientation of the scatterers. The velocities $v_{i}(\mathbf{r})$ generated in the fluid by a propagating sound wave are calculated from the Navier-Stokes equation. Then, the rate of dissipation of acoustic energy is obtained by integration of velocity gradients $\left(\partial v_{i} / \partial x_{k}\right)^{2}$ over the whole volume of occupied be viscous fluid [1]. In this microscopic approach the dissipative losses in the bulk and near the solid-fluid interfaces are correctly accounted for. Homogenization theory for phononic crystals of circular cylinders with low filling fraction in a viscous fluid was developed in Ref. [7]. It was shown that in the lowest approximation the effective mass density and bulk modulus are independent of viscosity. However, viscosity strongly reduces the scattering cross section of a homogenized cluster of solid cylinders.

Here the decay coefficient $\gamma_{\mathrm{ph}}$ for a 2D phononic crystal is calculated in the low-frequency limit $\omega, k \rightarrow 0$. In this limit the dispersion of sound is linear, $\omega=c_{\text {eff }} k$. The effective speed of sound $c_{\text {eff }}(\hat{\mathbf{k}})$ is obtained by calculating small (over $\left.k a_{0} \ll 1\right)$ corrections originating due to weak modulation of the plane wave solution by periodic scatterers [13]. It is represented by infinite sum over the reciprocal lattice vectors $\mathbf{G}$. In general case, $c_{\text {eff }}$ depends on the direction of propagation $\hat{\mathbf{k}}=\mathbf{k} / k$.

Propagating sound waves generate vibrations of pressure $p(\mathbf{r}) \exp (-i \omega t)$ and velocity $\mathbf{v}(\mathbf{r}) \exp (-i \omega t)$. Within the boundary layer $\delta$, velocity in a viscous fluid decays exponentially from its value in the bulk to zero at the interface of a motionless hard scatterer. The acoustic power (per unit length of a cylinder) dissipated around a hard scatterer is given by the following contour integral [1]:

$$
\dot{Q}=\frac{1}{2 \sqrt{2}} \sqrt{\rho \omega \eta} \oint_{l_{0}}|\mathbf{v}(\mathbf{r})|^{2} d l .
$$

Integration runs over contour $l_{0}$, which is the circumference of the scatterer. This formula is applied for calculation of dissipated power within a unit cell of $2 \mathrm{D}$ phononic crystal provided that viscous losses in the bulk, associated with viscosity coefficient $\xi$, are neglected. It is valid if the interface can be considered as flat within the lengths $\sim \delta$, i.e., $\delta \ll$ $L_{0}$, where $L_{0}$ is the length of the contour $l_{0}$ that separates fluid from solid. This inequality, together with the condition of homogenization, $k a_{0} \ll 1$, defines the frequency interval where the proposed theory is valid,

$$
\frac{\eta}{\rho L_{0}^{2}} \ll \omega \ll \frac{c_{\text {eff }}}{a_{0}} .
$$

For solid rods with $L_{0} \geqslant 1 \mathrm{~mm}$ in water environment ( $\eta=$ $0.01 \mathrm{~g} / \mathrm{cm} \mathrm{s}$ ) this inequality is satisfied starting from frequency $\sim 1 \mathrm{~Hz}$. Since $\dot{Q} \propto \sqrt{\eta}$, and keeping in mind the lowest approximation over viscosity $\eta$, the integrand $\mathbf{v}^{2}(\mathbf{r})$ can be calculated for ideal (inviscid) fluid. The energy losses due to temperature oscillations are omitted in Eq. (2) since for liquids like water they are negligible.

The decay coefficient for a wave propagating in a homogeneous dissipative medium is given by the ratio

$$
\gamma_{\mathrm{ph}}=\frac{\dot{Q}}{2 c_{\mathrm{eff}} E_{c}} .
$$

For the case of a homogenized phononic crystal $E_{c}$ is the acoustic energy within the unit cell

$$
E_{c}=\frac{1}{2} \int_{A_{c}} \rho(\mathbf{r})|\mathbf{v}(\mathbf{r})|^{2} d a \approx \frac{\rho}{2} \int_{A_{f}}|\mathbf{v}(\mathbf{r})|^{2} d a .
$$

The first integral in Eq. (5) is taken over the unit cell $A_{c}=$ $A_{s}+A_{f}$, containing the areas $A_{s}=f A_{c}$ and $A_{f}=(1-f) A_{c}$ occupied by solid and fluid, respectively. In the second integral the contribution of solid is neglected since Eq. (2) is already written in the approximation of hard scatterer. In what follows, we assume that the conditions of high acoustic 
contrast are satisfied [31],

$$
\lambda_{s} \gg \lambda, \quad \rho_{s} \gg \rho .
$$

Here $\lambda\left(\lambda_{s}\right)$ and $\rho\left(\rho_{s}\right)$ are the elastic modulus and density of the fluid (solid cylinder).

Pressure and velocity distributions in the low-frequency limit. Pressure $p(\mathbf{r})$ [and velocity $\mathbf{v}(\mathbf{r})=\nabla p(\mathbf{r}) / i \omega \rho(\mathbf{r})$ ] in a periodic system is represented by a Bloch wave

$$
p(\mathbf{r})=p_{0} e^{i \mathbf{k} \cdot \mathbf{r}}+\sum_{\mathbf{G} \neq 0} p_{k}(\mathbf{G}) e^{i(\mathbf{k}+\mathbf{G}) \cdot \mathbf{r}} .
$$

In the limit $\omega, k \rightarrow 0$ the first term is a homogenized plane wave of amplitude $p_{0}$ and the sum over $\mathbf{G}$ is a linear over $\omega$ (and $k$ ) correction due to weak modulation by a periodic set of scatterers. The set of linear equations for the Fourier components $p_{k}(\mathbf{G})$ follows from the wave equation for pressure in an inhomogeneous elastic medium [32]. In zero approximation over viscosity the set is written as follows:

$$
\begin{aligned}
& \sum_{\mathbf{G}^{\prime}} v\left(\mathbf{G}-\mathbf{G}^{\prime}\right)(\mathbf{k}+\mathbf{G}) \cdot\left(\mathbf{k}+\mathbf{G}^{\prime}\right) p_{k}\left(\mathbf{G}^{\prime}\right) \\
& \quad=\omega^{2} \sum_{\mathbf{G}^{\prime}} \beta\left(\mathbf{G}-\mathbf{G}^{\prime}\right) p_{k}\left(\mathbf{G}^{\prime}\right)
\end{aligned}
$$

Here $v(\mathbf{G})$ and $\beta(\mathbf{G})$ are the Fourier coefficients of the periodic function $1 / \rho(\mathbf{r})$ and $1 / \lambda(\mathbf{r})$. In the limit $\omega, k \rightarrow 0$ one obtains from Eq. (8) the following result for $p_{k}(\mathbf{G})$ [13]:

$$
p_{k}(\mathbf{G})=-p_{0} \sum_{\mathbf{G}^{\prime}}\left(\mathbf{k} \cdot \mathbf{G}^{\prime}\right) v\left(\mathbf{G}^{\prime}\right) I\left(\mathbf{G}, \mathbf{G}^{\prime}\right) .
$$

Here $I\left(\mathbf{G}, \mathbf{G}^{\prime}\right)=\left[\mathbf{G} \cdot \mathbf{G}^{\prime} v\left(\mathbf{G}-\mathbf{G}^{\prime}\right)\right]^{-1}$ implies inversion of the matrix which has the following elements:

$$
\begin{array}{ll}
\left(\rho_{s}^{-1}-\rho^{-1}\right) \mathbf{G} \cdot \mathbf{G}^{\prime} F\left(\mathbf{G}-\mathbf{G}^{\prime}\right), & \mathbf{G} \neq \mathbf{G}^{\prime}, \\
\left(\frac{f}{\rho_{s}}+\frac{1-f}{\rho}\right) G^{2}, & \mathbf{G}=\mathbf{G}^{\prime} .
\end{array}
$$

Substitution of Eq. (9) into Eq. (7) gives the distribution of pressure $p(\mathbf{r})$. Then the distribution of velocity $\mathbf{v}(\mathbf{r})=$ $\nabla p(\mathbf{r}) / i \omega \rho(\mathbf{r})$ can be easily calculated

$$
\mathbf{v}(\mathbf{r})=\frac{1}{\rho(\mathbf{r})}\left[\frac{p_{0} e^{i \mathbf{k} \cdot \mathbf{r}} \hat{\mathbf{k}}}{c_{\mathrm{eff}}(\hat{\mathbf{k}})}+\frac{1}{\omega} \sum_{\mathbf{G}} \mathbf{G} p_{k}(\mathbf{G}) e^{i \mathbf{G} \cdot \mathbf{r}}\right] .
$$

Here $c_{\text {eff }}(\hat{\mathbf{k}})=\lim _{k \rightarrow 0}(\omega / k)$ is the speed of low-frequency sound calculated in Ref. [13]

$$
\begin{aligned}
& c_{\mathrm{eff}}^{2}(\hat{\mathbf{k}}) \\
& =\frac{1}{\bar{\beta}}\left[\bar{\nu}-\sum_{\mathbf{G}, \mathbf{G}^{\prime} \neq 0}(\hat{\mathbf{k}} \cdot \mathbf{G})\left(\hat{\mathbf{k}} \cdot \mathbf{G}^{\prime}\right) v(\mathbf{G}) v\left(-\mathbf{G}^{\prime}\right) I\left(\mathbf{G}^{\prime}, \mathbf{G}\right)\right], \\
& \bar{v}=\frac{f}{\rho_{s}}+\frac{1-f}{\rho}, \quad \bar{\beta}=\frac{f}{\lambda_{s}}+\frac{1-f}{\lambda} .
\end{aligned}
$$

Note that while this result is obtained in the low-frequency limit, the periodicity of the structure is strongly manifested through the sums over the reciprocal-lattice vectors G. Application of Eq. (12) to a medium without periodicity (a set of disordered scatterers or porous medium) is not justified and may lead to essential error.
Distributions of pressure and velocity given by Eqs. (7) and (11) calculated in the low-frequency limit are principally different. Pressure, being a continuous function at the fluid-solid interface, is reduced to a plane wave with wavelength $2 \pi / k \gg$ $a_{0}$ weakly modulated by a function having the period of the lattice, Eqs. (7) and (9). Unlike this, the velocity (11) does not homogenize. Both terms in Eq. (11) exhibit periodicity of the lattice. Since the velocity distribution is calculated for inviscid fluid its tangential component does not match the velocity of vibrating solid inclusion. The discontinuity of the tangential component of $\mathbf{v}(\mathbf{r})$ does not allow homogenization of velocity as a vector. At the same time, exactly this discontinuous component which does not vanish at the fluid-solid boundary gives the contribution to the the integral (2). The continuous normal component becomes negligible at the boundary, provided the condition of high acoustic contrast (6) is satisfied. Note, that in a viscous fluid, the vector of velocity homogenizes due to a no-slip boundary condition.

Homogenization of pressure occurs for any elastic material of the cylinders with density $\rho_{s}$ except $\rho_{s}=\infty$. Sound waves do not penetrate in a material with infinite density. Therefore, $p(\mathbf{r})=0$ inside or on the surface of hard scatterers. At the same time for any point in the fluid pressure remains constant, $p(\mathbf{r}) \approx p_{0}$, if $k \rightarrow 0$. Thus, pressure suffers a discontinuous jump at each interface. Since the discontinuous jumps have the periodicity of the lattice, the Fourier coefficients $p_{k}(\mathbf{G})$ do not vanish in the limit $k \rightarrow 0$. According to Eq. (9) $p_{k}(\mathbf{G}) \sim k$. For this Fourier coefficients to remain finite at $k \rightarrow 0$ the matrix $I\left(\mathbf{G}, \mathbf{G}^{\prime}\right)$ must be singular in the limit $\rho_{s} \rightarrow \infty$ [33]. To avoid singularity in numerical inversion of the matrix $\left[\mathbf{G} \cdot \mathbf{G}^{\prime} v\left(\mathbf{G}-\mathbf{G}^{\prime}\right)\right]$ the density $\rho_{s}$ must significantly exceed $\rho$ but remain finite. For any strong but finite acoustic contrast, the pressure is a continuous function allowing homogenization. However, the acoustic energy accumulated inside the solid cylinders can be neglected due to inequality (6).

Once the distribution of velocity is known from Eqs. (11) and (9), the dissipated power Eq. (2) and acoustic energy within the unit cell Eq. (5) can be calculated. Then the decay coefficient is obtained from Eq. (4). Simple but cumbersome calculations lead to the following result:

$$
\gamma_{\mathrm{ph}}(\hat{\mathbf{k}})=\frac{L_{0}}{2 A_{c} c_{\mathrm{eff}}(\hat{\mathbf{k}})} \sqrt{\frac{\omega \eta}{2 \rho}} \frac{M(\hat{\mathbf{k}})}{N(\hat{\mathbf{k}})} .
$$

Here the quantities $M(\hat{\mathbf{k}})$ and $N(\hat{\mathbf{k}})$ are complicated functionals of the form factors $F(\mathbf{G})$ and $L(\mathbf{G})$,

$$
\begin{aligned}
M(\hat{\mathbf{k}})= & 1+\frac{2}{\rho} \sum_{\mathbf{G}, \mathbf{G}^{\prime}} L^{*}(\mathbf{G})(\hat{\mathbf{k}} \cdot \mathbf{G})\left(\hat{\mathbf{k}} \cdot \mathbf{G}^{\prime}\right) F\left(\mathbf{G}^{\prime}\right) I\left(\mathbf{G}, \mathbf{G}^{\prime}\right) \\
& +\frac{1}{\rho^{2}} \sum_{\mathbf{G}_{\mathbf{1}}, \ldots, \mathbf{G}_{\mathbf{4}}} L^{*}\left(\mathbf{G}_{\mathbf{1}}+\mathbf{G}_{\mathbf{3}}\right)\left(\mathbf{G}_{\mathbf{1}} \cdot \mathbf{G}_{\mathbf{3}}\right)\left(\hat{\mathbf{k}} \cdot \mathbf{G}_{\mathbf{2}}\right)\left(\hat{\mathbf{k}} \cdot \mathbf{G}_{\mathbf{4}}\right) \\
& \times F\left(\mathbf{G}_{\mathbf{2}}\right) F\left(\mathbf{G}_{\mathbf{4}}\right) I\left(\mathbf{G}_{\mathbf{1}}, \mathbf{G}_{\mathbf{2}}\right) I\left(\mathbf{G}_{\mathbf{3}}, \mathbf{G}_{\mathbf{4}}\right), \\
N(\hat{\mathbf{k}})= & 1-f-\frac{2}{\rho} \sum_{\mathbf{G}, \mathbf{G}^{\prime}} \hat{\mathbf{k}} \cdot \mathbf{G} \hat{\mathbf{k}} \cdot \mathbf{G}^{\prime} F^{*}(\mathbf{G}) F\left(\mathbf{G}^{\prime}\right) I\left(\mathbf{G}, \mathbf{G}^{\prime}\right) \\
& -\frac{1}{\rho^{2}} \sum_{\mathbf{G}_{\mathbf{1}}, \ldots, \mathbf{G}_{\mathbf{4}}} F^{*}\left(\mathbf{G}_{\mathbf{1}}+\mathbf{G}_{\mathbf{3}}\right)\left(\mathbf{G}_{\mathbf{1}} \cdot \mathbf{G}_{\mathbf{3}}\right)\left(\hat{\mathbf{k}} \cdot \mathbf{G}_{\mathbf{2}}\right)\left(\hat{\mathbf{k}} \cdot \mathbf{G}_{\mathbf{4}}\right) \\
& \times F\left(\mathbf{G}_{\mathbf{2}}\right) F\left(\mathbf{G}_{\mathbf{4}}\right) I\left(\mathbf{G}_{\mathbf{1}}, \mathbf{G}_{\mathbf{2}}\right) I\left(\mathbf{G}_{\mathbf{3}}, \mathbf{G}_{\mathbf{4}}\right) .
\end{aligned}
$$




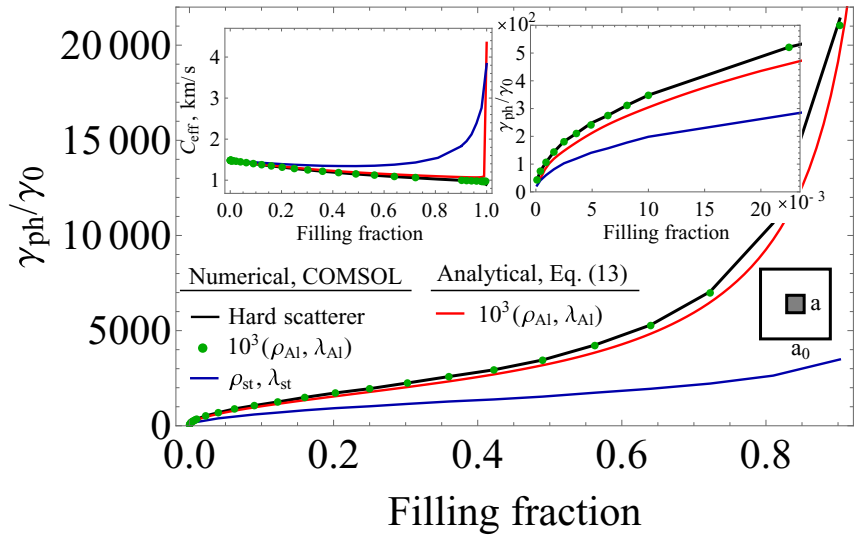

FIG. 1. Normalized decay coefficient of sound propagating in isotropic square lattice with square cross section of the cylinders in viscous water background as a function of filling fraction $f=$ $\left(a / a_{0}\right)^{2}, a_{0}=5.5 \mathrm{~mm}$. Left inset: Speed of sound (in $\mathrm{m} / \mathrm{s}$ ) given by Eq. (12). Right inset: Blowup of the region of low $f$, where $\gamma_{\mathrm{ph}} \sim$ $\sqrt{f}$. The decay coefficient calculated from Eq. (13) with 2028 plane waves is shown by red curve. The parameters of the scatterers are selected to be $10^{3}$ of those of aluminum: $\rho_{s}=10^{3} \times 2.7 \mathrm{~g} / \mathrm{cm}^{3}, \lambda_{s}=$ $10^{3} \times 51 \mathrm{GPa}$, and $c_{s}=\sqrt{\lambda_{s} / \rho_{s}}=4346 \mathrm{~m} / \mathrm{s}$. Numerical results obtained by COMSOL for the same elastic parameters are shown by green dots. Black line shows the results obtained by COMSOL for hard scatterers, i.e., applying the condition of zero velocity, $\mathbf{v}(\mathbf{r})=0$, for the cylinders. Blue curve is the decay coefficient of stainless steel calculated by COMSOL.

The linear form factor $L(\mathbf{G})$ is defined as the integral over the circumference of the solid scatterer,

$$
L(\mathbf{G})=\frac{1}{L_{0}} \oint_{l_{0}} e^{-i \mathbf{G} \cdot \mathbf{r}} d l .
$$

The exact result (13) confirms the estimate (1) up to the dimensionless factor $M(\hat{\mathbf{k}}) / N(\hat{\mathbf{k}})$. This factor takes into account the microstructure of the phononic crystal and the details of the formation of the boundary layer. It turns out that depending on geometry of the unit cell this numerical factor can be as big as $\sim 10-10^{3}$. While the scaling of the decay coefficient (1) with $\omega$ and $\eta$ is valid for a nonperiodic set of cylinders, the exact result (13) is applicable only for a periodic structure.

Decay coefficient for isotropic and anisotropic lattices. We apply the obtained results for phononic crystal with square lattice. If the scatterers have the same or higher symmetry, then this structure is isotropic in the long-wavelength limit. If, however, the scatterers are of lower symmetry, then the structure exhibits anisotropy. Two lattices with square and rectangular cross sections of the scatterers will be considered in this section.

The decay coefficient given by Eq. (13) is plotted in Fig. 1 versus filling fraction for a square lattice with a square cross section of the scatterers at frequency $\omega / 2 \pi=50 \mathrm{kHz}$, which is well below the fundamental band gap. The results obtained from Eq. (13) are compared to the decay coefficient numerically calculated by COMSOL. The decay coefficient $\gamma_{\mathrm{ph}}$ is obtained as imaginary part of the numerically calculated $k=k(\omega)$ for the lowest transmission band, assuming that $\omega$ is real. For this isotropic lattice $\gamma_{\mathrm{ph}}$ is independent of $\hat{\mathbf{k}}$.
Black line is obtained by COMSOL for the structure of cylinders in viscous water which do not vibrate under a passing sound wave (hard scatterers). The limit of hard scatterers is well-approximated by green dots obtained by COMSOL for an elastic material with $\rho_{s}=10^{3} \rho_{\mathrm{Al}}$ and $\lambda_{s}=10^{3} \lambda_{\mathrm{Al}}$. This hypothetic material possesses practically infinite acoustic impedance, $Z=10^{3} Z_{\mathrm{Al}}$, i.e., it behaves like a hard scatterer. At the same time, speed of longitudinal sound in it is the same as for aluminum, $c_{s}=\sqrt{\lambda_{s} / \rho_{s}}=4346 \mathrm{~m} / \mathrm{s}$. Therefore, the effective speed of sound in the phononic crystal must approach $4346 \mathrm{~m} / \mathrm{s}$ in the limit $f \rightarrow 1$. As seen in the left insert the effective speed of sound gradually decreases with $f$ but near $f=1$ there is a sharp turn toward the value of 4346 $\mathrm{m} / \mathrm{s}$ (red curve). This sharp turn becomes a singularity in the limiting case of hard scatterers (black curve). The speed of sound and the decay coefficient calculated from Eq. (13) for the same material parameters (red curve) are close to the numerical result obtained by COMSOL. Right insert shows the region of low filling fractions. At $f \ll 1$, the decay coefficient grows fast. The square-root dependence, $\gamma_{\mathrm{ph}} \sim \sqrt{f}$ predicted by Eq. (1) is reproduced. Since the numerical and analytical results obtained for phononic crystal of hard scatterers are in excellent agreement we may conclude that the approximations made in derivation of the decay coefficient (13) are justified.

If the condition of high acoustic contrast (6) is weakened, then the decay coefficient becomes smaller due to partial penetration of acoustic energy inside scatterers. Also near vibrating solid surface the gradients of velocity within the viscous boundary layer are reduced that leads to lower viscous dissipation. Blue curve in Fig. 1 represents COMSOL result obtained for stainless steel cylinders. For this structure the decay coefficient turns out to be essentially less than for hard scatterers. The ratio of impedances $Z_{\text {steel }} / Z_{\text {water }} \approx 30$ is not sufficiently large to justify hard scatterers approximation. The difference between the results obtained for hard scatterers (black curve in Fig. 1) and stainless steel scatterers (blue curve) strongly increases for $f>0.8$. The spacings between the cylinders become narrow and in the case of hard scatterers sound propagates through narrow fluid channels. Strongly enhanced viscosity of air in narrow slits formed by aluminum plates was recently observed in Ref. [6]. Aluminum objects in air behave like hard scatterers since $Z_{\mathrm{Al}} / Z_{\mathrm{air}} \sim 10^{4}$. Therefore, the observed enhancement of thermoviscous dissipation of sound is of the same nature as fast grows of the decay coefficient shown in Fig. 1. This effect was explored in design of phononic black hole [34].

The proposed approach becomes invalid if the viscous boundary layers of two neighboring scatterers overlap. This occurs near $f=1$. In water the thickness $\delta \approx 3$ micron at frequency $50 \mathrm{kHz}$. For the lattice in Fig. 1 with period $a_{0}=$ $5.5 \mathrm{~mm}$ the viscous layers overlap for $f>0.98$. Within the region of fillings corresponding to practical applications of phononic crystals, $0.1<f<0.8$, the decay length of sound is reduced by $10^{3}-10^{4}$ times as compared to that in free water.

It is known that phononic crystals with anisotropic unit cell behave like metafluids with anisotropic dynamic mass [18-22,35,36]. Viscosity, being a dynamical property also turns out to be anisotropic. Using Eq. (13) the parameters of a structure with desired anisotropy of viscous losses can be calculated. A metamaterial with anisotropic viscosity is 


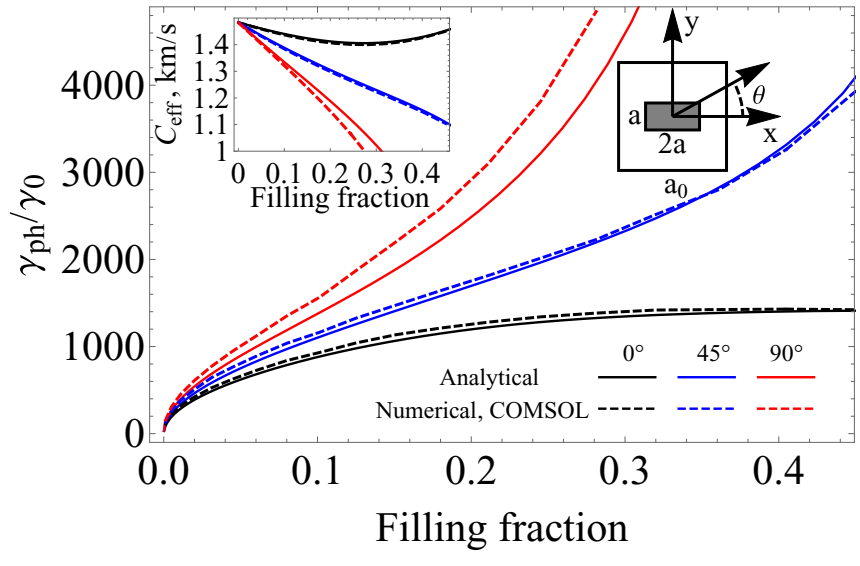

FIG. 2. The same as in Fig. 1 for a square lattice of anisotropic hard scatterers with rectangular cross section. The parameters of the scatterers are $10^{3} \rho_{s}$ and $10^{3} \lambda_{s}$. COMSOL results are in good agreement with the proposed theory for $\theta=0^{\circ}$ and $\theta=45^{\circ}$. A noticeable difference for the direction $\theta=90^{\circ}$ is explained by much higher dissipation.

necessary for engineering of a recently proposed hydrodynamic cloak [37]. Anisotropy of viscous losses was experimentally demonstrated in Refs. [36,38].

Figure 2 shows the decay coefficient calculated for phononic crystal with square unit cell and anisotropic scatterers. Each scatterer has a rectangular cross section with sides in the ratio $1: 2$, as shown in the insert. The decay coefficient is calculated using formula (13) (solid curves) at $\omega / 2 \pi=$ $50 \mathrm{kHz}$ and confirmed by numerical COMSOL simulations (dashed curves) for three directions of propagation. The results can be easily rescaled for other frequencies since in the region of homogenization $\gamma_{\mathrm{ph}} \sim \sqrt{\omega}$.

Higher losses are obtained for sound wave propagating along the shorter side of the rectangle $\left(\theta=90^{\circ}\right)$. This result sounds counterintuitive since the longer viscous boundary layer is formed for sound propagating along the longer side $\left(\theta=0^{\circ}\right)$. However, the length of this layer is not the only factor. Local dissipation is defined by velocity gradients which strongly enhanced near sharp corners. The number of corners the wave meets per unit of length is larger for $\theta=90^{\circ}$ than for $\theta=0^{\circ}$. Another important factor is the effective speed of sound $c_{\text {eff }}$ that appears in the denominator of Eq. (13). Left insert to Fig. 2 shows much lower speed of sound for $\theta=$ $90^{\circ}$. Slower sound is in line with previously reported [21,35] higher inertia along the direction $\theta=90^{\circ}$. Note that for $1 \mathrm{D}$ layered system the factor of long viscous layers dominates and the factor of multiple corners along the sound path is absent. This leads to higher dissipation of sound propagating along the layers [36].

Dissipation always slows down propagation of sound. This can be seen in the insert to Fig. 2. For the directions of $0^{\circ}$ and $45^{\circ}$ the dissipation is low, therefore the difference in the speed of sound calculated from Eq. (12) for inviscid fluid and using COMSOL for viscous water is relatively small. However, dissipation is essentially increased for the direction of $90^{\circ}$ that leads to noticeable reduction of the effective speed of sound. It can be seen that two red curves in the insert linearly diverge with filling fraction, i.e., the correction to the dispersion law caused by dissipation is proportional to $f$. It is well known that pure imaginary perturbation to Hamiltonian leads to quadratic correction in the dispersion law (or to quadratic shift of energy levels in quantum system, while the broadening is linear). Since according to Eq. (1) the relative dissipative perturbation $\Delta E / E \sim \sqrt{f}$, the corresponding quadratic correction to the dispersion law grows linearly with $f$.

Calculated decay coefficient for the arrays of hard scatterers in Figs. 1 and 2 represents the maximum possible for a given lattice (and fluid viscosity) level of sound absorption. For the same structures of real solid scatterer the decay coefficient is lower due to vibration of the scatterers.

Viscous losses in free water grow quadratically with frequency, $\gamma_{0} \sim \omega^{2}$. In phononic crystals, the losses grow as $\gamma_{\mathrm{ph}} \sim \sqrt{\omega}$. At frequency $\omega^{\star} \sim\left(c^{2} / a\right)^{2 / 3}(f \rho / \eta)^{1 / 3}$ viscous losses in the bulk and in the boundary layers become comparable. For a phononic crystal with $a_{0}=5.5 \mathrm{~mm}$ and $f=$ 0.5 embedded in water this frequency is about $1 \mathrm{MHz}$. Of course, at such high frequency the phononic crystal cannot be considered as a homogeneous medium, therefore the formula for $\omega^{\star}$ is only a rough estimate.

Any inhomogeneities at the circumference of the scatterers, like bumps, indents, or angles produce strong gradients in distribution of fluid velocities that increase viscous losses. High losses strongly limit observation of resonances leading to negative effective mass and/or elastic modulus. Recently, the role of viscothermal losses in periodic elastic structures has been experimentally studied in Refs. $[5,6,11,39]$. It was demonstrated that losses do not allow observation of some effects predicted in the approximation of inviscid medium. In particular, negative refraction predicted for 2D periodic structure in the lossless limit [40] and resonant transmission through an array of narrow slits [6] are suppressed by thermoviscous damping. On the other hand, viscous losses bring new properties to acoustic metamaterials which can be useful if appropriately tailored and explored.

Conclusions. In conclusion, we have developed a microscopic theory of sound decay due to viscous losses in a phononic crystal of rigid cylinders embedded in viscous fluid. Analytic result is derived for the decay coefficient of sound propagating in the low-frequency limit when phononic crystal behaves like a homogeneous fluid. This homogeneous fluid exhibits anisotropic viscous losses if the unit cell is asymmetric. The level of anisotropy and decay coefficient grow fast with filling fraction. Formation of viscous boundary layers around solid cylinders leads to enhancement of viscous losses by two to four orders of magnitude as compared to free water. Analytical results are obtained for arbitrary 2D Bravais lattice with rigid cylinders. Comparison with numerical COMSOL calculations demonstrates good agreement for phononic crystals with high acoustic contrast between the constituents. Decay coefficient is reduced for lower acoustic contrast. Continuously varying anisotropy of viscous losses represents a new property of phononic crystal as a viscous metamaterial. This property will find new applications, for example in engineering of dissipative hydrodynamic cloak and acoustic black hole.

Acknowledgments. This work is supported by an EFRI grant from the National Science Foundation (Grant No. 
1741677) and by CONACyT (Mexico) through Grant No. A1-S-23120. A.A.K. is thankful for hospitality at the Instituto de Física Universidad Autónoma de Puebla where part of this work has been accomplished.
[1] L. D. Landau and E. M. Lifshitz, Fluid Mechanics, 2nd ed. (Elsevier, Oxford, 1984).

[2] B. P. Konstantinov, To the problem of sound absorption at reflection from a solid boundary, Zh. Tekh. Fiz. 9, 226 (1939).

[3] F. F. Legusha, The Konstantinov effect and sound absorption in inhomogeneous media, Sov. Phys. Uspek. 27, 887 (1984).

[4] L. M. Brekhovskikh and O. A. Godin, Acoustics of Layered Media I, 2nd ed. (Springer, Berlin, 1998).

[5] Xue Jiang, Yong Li, and Likun Zhang, Thermoviscous effects on sound transmission through a metasurface of hybrid resonances, J. Acoust. Soc. Am. 141, EL363 (2017).

[6] G. P. Ward, R. K. Lovelock, A. R. J. Murray, A. P. Hibbins, J. R. Sambles, and J. D. Smith, Boundary-Layer Effects on Acoustic Transmission through Narrow Slit Cavities, Phys. Rev. Lett. 115, 044302 (2015).

[7] E. Reyes-Ayona, D. Torrent, and J. Sánchez-Dehesa, Homogenization theory for periodic distributions of elastic cylinders embedded in a viscous fluid, J. Acoust. Soc. Am. 132, 2896 (2012).

[8] K. C. Nunan and J. B. Keller, Effective viscosity of a periodic suspension, J. Fluid Mech. 142, 269 (1984).

[9] For 3D phononic crystal $\gamma_{\mathrm{ph}} \sim f^{2 / 3}$ and for a layered system $\gamma_{\mathrm{ph}} \sim f^{0}$. For a $D$-dimensional arrangement of scatterers $\gamma_{\mathrm{ph}} \sim$ $f^{(D-1) / D}$.

[10] W. H. Lin and A. C. Raptis, Acoustic scattering by elastic solid cylinders and spheres in viscous fluids, J. Acoust. Soc. Am. 73, 736 (1983).

[11] M. Molerón, M. Serra-Garcia, and C. Daraio, Visco-thermal effects in acoustic metamaterials: from total transmission to total reflection and high absorption, New J. Phys. 18, 033003 (2016).

[12] M. Kafesaki, R. S. Penciu, and E. N. Economou, Air Bubbles in Water: A Strongly Multiple Scattering Medium for Acoustic Waves, Phys. Rev. Lett. 84, 6050 (2000).

[13] A. A. Krokhin, J. Arriaga, and L. N. Gumen, Speed of Sound in Periodic Elastic Composites, Phys. Rev. Lett. 91, 264302 (2003).

[14] Z. Hou, F. Wu, X. Fu, and Y. Liu, Effective elastic parameters of the two-dimensional phononic crystal, Phys. Rev. E 71, 037604 (2005).

[15] Qing Ni and Jianchun Cheng, Anisotropy of effective velocity for elastic wave propagation in two-dimensional phononic crystals at low frequencies, Phys. Rev. B 72, 014305 (2005).

[16] J. Mei, Z. Liu, W. Wen, and P. Sheng, Effective Mass Density of Fluid-Solid Composites, Phys. Rev. Lett. 96, 024301 (2006).

[17] D. Torrent and J. Sánchez-Dehesa, Effective parameters of clusters of cylinders embedded in a nonviscous fluid or gas, Phys. Rev. B 74, 224305 (2006).

[18] D. Torrent and J. Sánchez-Dehesa, Anisotropic mass density by two-dimensional acoustic metamaterials, New J. Phys. 10, 023004 (2008).

[19] A. N. Norris, Acoustic metafluids, J. Acoust. Soc. Am. 125, 839 (2009).
[20] R. V. Craster, J. Kaplunov, and A. V. Pichugin, High-frequency homogenization for periodic media, Proc. R. Soc. A 466, 2341 (2010).

[21] L. N. Gumen, J. Arriaga, and A. A. Krokhin, Metafluid with anisotropic dynamic mass, Low Temp. Phys. 37, 975 (2011); Y. Zubov, B. Djafari-Rouhani, and A. Krokhin, Dynamical effective parameters of elastic superlattice with strong acoustic contrast between the constituents, ibid. 44, 1280 (2018).

[22] A. N. Norris, A. L. Shuvalov, and A. A. Kutsenko, Analytical formulation of three-dimensional dynamic homogenization for periodic elastic composites, Proc. Roy. Soc. A 468, 1629 (2012).

[23] J. Flores-Méndez and F. Pérez-Rodríguez, Metasolid with anisotropic mass density, Europhys. Lett. 103, 54001 (2013).

[24] D. Torrent, Y. Pennec, and B. Djafari-Rouhani, Effective medium theory for elastic metamaterials in thin elastic plates, Phys. Rev. B 90, 104110 (2014).

[25] A. Konovalenko, E. Gutiérrez-Reyes, A. L. González, J. FloresMéndez, and F. Pérez-Rodríguez, Nonlocal metasolid response of homogenized phononic crystals, J. Appl. Phys. 121, 155102 (2017).

[26] A. A. Krokhin and P. Halevi, Influence of weak dissipation on the photonic band structure of periodic composites, Phys. Rev. B 53, 1205 (1996).

[27] E. Psarobas, Viscoelastic response of sonic band-gap materials, Phys. Rev. B 64, 012303 (2001).

[28] M. I. Hussein, Theory of damped Bloch waves in elastic media, Phys. Rev. B 80, 212301 (2009).

[29] R. P. Moiseyenko and V. Laude, Material loss influence on the complex band structure and group velocity in phononic crystals, Phys. Rev. B 83, 064301 (2011).

[30] M. I. Hussein and M. J. Frazier, Damped phononic crystals and acoustic metamaterials, in Acoustic Metamaterials and Phononic Crystals, Springer Series in Solid-State Sciences 173, edited by P. A. Deymier (Springer-Verlag, Berlin, 2013).

[31] The results for a set of periodic cylinders of finite elasticity will be published elsewhere.

[32] M. S. Kushwaha, P. Halevi, L. Dobrzynski, and B. DjafariRouhani, Acoustic Band Structure of Periodic Elastic Composites, Phys. Rev. Lett. 71, 2022 (1993).

[33] Singularity of the inverse matrix impies that the direct matrix $\left[\mathbf{G} \cdot \mathbf{G}^{\prime} v\left(\mathbf{G}-\mathbf{G}^{\prime}\right)\right]$ is also singular, i.e., its determinat vanishes when $k \rightarrow 0$.

[34] A. Climente, D. Torrent, and J. Sánchez-Dehesa, Omnidirectional broadband acoustic absorber based on metamaterials, Appl. Phys. Lett. 100, 144103 (2012).

[35] L. Zigoneanu, B. I. Popa, A. F. Starr, and S. A. Cummer, Design and measurements of a broadband 2D acoustic metamaterial with anisotropic mass density, J. Appl. Phys. 109, 054906 (2011).

[36] M. D. Guild, V. M. García-Chocano, W. Kan, and J. SánchezDehesa, Acoustic metamaterial absorbers based on multilayered sonic crystals, J. Appl. Phys. 117, 114902 (2015). 
[37] J. Park, Jae Ryoun Youn, and Young Seok Song, Hydrodynamic Metamaterial Cloak for Drag-Free Flow, Phys. Rev. Lett. 123, 074502 (2019).

[38] E. Walker, A. Neogi, A. Bozhko, Yu. Zubov, J. Arriaga, H. Heo, J. Ju, and A. A. Krokhin, Nonreciprocal Linear Transmission of Sound in a Viscous Environment with Broken P Symmetry, Phys. Rev. Lett. 120, 204501 (2018).
[39] V. Cutanda Henríquez, V. M. García-Chocano, and J. SánchezDehesa, Viscothermal losses in double-negative acoustic metamaterials, Phys. Rev. Appl. 8, 014029 (2017).

[40] R. Graciá-Salgado, V. García-Chocano, D. Torrent, and J. Sánchez-Dehesa, Negative mass density and density-nearzero quasi-two-dimensional metamaterial: Design and applications, Phys. Rev. B 88, 224305 (2013). 